\title{
Cryptogram Decoding for OCR using Numerization Strings
}

\author{
Gary Huang, Erik Learned-Miller, Andrew McCallum \\ University of Massachusetts \\ Department of Computer Science \\ 140 Governors Drive, Amherst, MA 01003 \\ \{ghuang, elm, mccallum\}@cs.umass.edu
}

\begin{abstract}
OCR systems for printed documents typically require large numbers of font styles and character models to work well. When given an unseen font, performance degrades even in the absence of noise. In this paper, we perform OCR in an unsupervised fashion without using any character models by using a cryptogram decoding algorithm. We present results on real and artificial OCR data.
\end{abstract}

\section{Introduction and Related Work}

Most OCR systems for machine-print text need to know about a large collection of font styles to work well. Such systems are font-dependent and suffer in accuracy when given documents printed in novel font styles. We examine an alternative approach that groups together similar characters in the document and solves a cryptogram to assign labels to clusters of characters. This method does not require any character models, so it is able to handle arbitrary fonts. It can take advantage of patterns such as regularities in image distortions that are particular to each document. In addition, the cryptogram decoding procedure is well-suited for performing OCR on images compressed using tokenbased methods such as Djvu, Silx, and DigiPaper.

Treating OCR as a cryptogram decoding problem dates back at least to papers by Nagy [10] and Casey [2] in 1986. More recently, Ho and Nagy [3] develop an unsupervised OCR system that performs character clustering followed by lexicon-based decoding. Their decoding procedure iteratively applies a set of rules to find mappings by comparing a "v/p" ratio against manually set thresholds. One major difference between their work and ours is that we use probabilistic reasoning instead of predefined thresholds. In [7], Lee presents a unified approach to decode substitution ciphers by using Hidden Markov Models and the expectation maximization algorithm. He uses n-gram statistics as model priors, whereas we use entire word patterns. Breuel [1] in- troduced a supervised OCR system that is font independent, but it does not take advantage of token-based image compression.

\section{The Model}

We take binary images of machine printed text as input, and we use a segmentation scheme that assumes each ink blot (i.e., connected component) is a separate character, though some effort is made to identify characters composed of multiple ink blots, such as those with accent symbols and the letters $i$ and $j$. An object defined in this manner can correspond to (1) exactly one character, (2) a partial character, or (3) multiple characters such as the ligatures $f i$ and $f f i$. These objects are then clustered using greedy agglomerative clustering, so that the input document is transformed into a string of cluster assignments in place of the actual characters. By examining the patterns of repetitions of cluster IDs and comparing them to the patterns for dictionary words, we decode the mapping between cluster IDs and characters in the output alphabet. We describe each step in more detail below.

\subsection{Character Clustering}

Two straightforward distance measures between binary images $A$ and $B$ are the Hamming and the Hausdorff distances. The Hamming distance is simply the number of pixels on which $A$ and $B$ differ. It is fast and easy to calculate, but is not robust to noise or variations like stroke thickness. Hausdorff distance [8] is defined as

$$
h(A, B)=\max _{a \in A} \min _{b \in B} d(a, b),
$$

where $d$ is a metric, such as the Euclidean distance. If the Hausdorff distance from $A$ to $B$ is $\delta$, then for every point $a \in A$, there is a point in $B$ within distance $\delta$. 
To reduce effects from noisy pixels on the distance, we "soften" the Hausdorff distance so that $h_{p}(A, B)=\delta$ means for at least $p$ percent of the points $a \in A$, there is a point in $B$ within distance $\delta$. To make the Hausdorff measure symmetric, we take the mean of $h_{p}(A, B)$ and $h_{p}(B, A)$. In our experiments, we use this average with $p=95$.

The Hausdorff measure is more robust than the Hamming measure, but is expensive to compute for the $O\left(n^{2}\right)$ pairwise distances, where $n$ is the number of images. We take advantage of the speed of the Hamming distance and the robustness of Hausdorff distance by using the canopy method devised by McCallum et al [9]. First, the Hamming distance is computed for all pairs of images, and two distance thresholds $T_{1}$ and $T_{2}$ are specified, where $T_{1}>T_{2}$. Next, we go through the list of images in any order and remove one image from the list to serve as the seed of a new canopy. All images in the list within distance $T_{1}$ of the seed image are placed into the new canopy, and all images within distance $T_{2}$ are removed from the list. This process is repeated until the list is empty. The more expensive Hausdorff measure is then used for pairwise distances within each canopy.

After all pairwise distances have been computed, the images are partitioned using hierarchical agglomerative clustering. Inter-cluster similarity is computed by the group average. I.e., the distance between clusters $G_{1}$ and $G_{2}$ is given by $d\left(G_{1}, G_{2}\right)=\frac{1}{\left|G_{1}\right| \cdot\left|G_{2}\right|} \sum_{A \in G_{1}} \sum_{B \in G_{2}} h(A, B)$. To choose the final number of clusters, we use the elbow criterion described in the experiments section.

\subsection{Character Decoding}

Consider the following encoding of an English word:

$$
\alpha \beta \gamma \gamma \beta \gamma \gamma \beta \delta \delta \beta
$$

If each Greek letter stands for one letter, what is the decoding? After some thought, it should be clear that it is the word "Mississippi," since no other English word has that particular pattern of letters.

For each word represented as a string of cluster assignments, we compute its numerization string by going from left to right, assigning 1 to the first cluster ID, 2 to the second distinct cluster ID, 3 to the third distinct cluster ID, etc. For the above string, suppose the cluster assignments are 73202032020317173 , then the corresponding numerization string is 12332332442 .

By computing the numerization strings for every document and dictionary word, we identify code words in the document that map to a unique dictionary word or a small number dictionary words. This gives an initial mapping between cluster IDs and output characters.
Formally, let $E=\left(e_{1}, e_{2}, \ldots, e_{n}\right)$ be the sequence of words encoded by cluster assignments, $C=\left\{c_{i}\right\}$ be the set of cluster IDs, and $\Sigma=\left\{\alpha_{j}\right\}$ be the alphabet of the target language. Our goal is to compute the set of assignments that maximizes $P\left(\left\{c_{i}=\alpha_{j}\right\} \mid E\right)$. By considering one mapping at a time, we write

$$
\begin{aligned}
P\left(c_{i}=\alpha_{j} \mid E\right) & =\frac{P\left(E \mid c_{i}=\alpha_{j}\right) P\left(c_{i}=\alpha_{j}\right)}{P(E)} \\
& \propto P\left(E \mid c_{i}=\alpha_{j}\right) P\left(c_{i}=\alpha_{j}\right) \\
& \propto P\left(e_{1}, e_{2}, \ldots, e_{n} \mid c_{i}=\alpha_{j}\right) \\
& \approx \prod_{k=1}^{n} P\left(e_{k} \mid c_{i}=\alpha_{j}\right) \\
& =\prod_{k=1}^{n} \frac{P\left(c_{i}=\alpha_{j} \mid e_{k}\right) P\left(e_{k}\right)}{P\left(c_{i}=\alpha_{j}\right)} \\
& \propto \prod_{k=1}^{n} P\left(c_{i}=\alpha_{j} \mid e_{k}\right),
\end{aligned}
$$

where we have applied the naive Bayes assumption, used Bayes' rule, and assumed a uniform prior for $P\left(c_{i}=\alpha_{j}\right)$.

The quantity $P\left(c_{i}=\alpha_{j} \mid e_{k}\right)$ is calculated by normalizing the count of the number of times cluster ID $c_{i}$ maps to output letter $\alpha_{j}$ among the dictionary words that have the same numerization string as $e_{k}$. We used Laplace smoothing with $\lambda=0.001$ to avoid zero probabilities.

Once $P\left(c_{i}=\alpha_{j} \mid E\right)$ has been calculated for every $c_{i}$ and $\alpha_{j}$, each cluster $c_{i}$ is mapped to character $\operatorname{argmax}_{\alpha_{j}} P\left(c_{i}=\alpha_{j} \mid E\right)$. Not all assignments will be correct at this point, because of words whose numerization strings don't have much discriminating power. We solve this problem by using the set of mappings of which we are confident to infer the less confident ones.

\subsection{Confidence Estimation}

An intuitive way to measure the confidence of an assignment for $c_{i}$ is to look at how peaky the distribution $P\left(c_{i}=\cdot \mid E\right)$ is. Entropy quantifies this measure. For every cluster ID $c_{i}$, the entropy of its assignment is

$$
H\left(c_{i}\right)=-\sum_{\alpha_{j} \in \Sigma} P\left(c_{i}=\alpha_{j} \mid E\right) \log \left(P\left(c_{i}=\alpha_{j} \mid E\right)\right) .
$$

Sorting the entropies in ascending order gives a list of $c_{i}$ 's whose assignments are in decreasing confidence. Recall that each code word $e_{k}$ is associated with a list of dictionary words $D_{k}$ that have the same numerization string. In general, some dictionary words in $D_{k}$ are incompatible with the mode of $P\left(c_{i}=\cdot \mid E\right)$. Our refinement strategy is to iterate the $c_{i}$ 's as sorted by entropy, assume the mapping of $c_{i}=\operatorname{argmax}_{\alpha_{j}} P\left(c_{i}=\alpha_{j} \mid E\right)$ to be true, and for each 
code word that contains $c_{i}$, remove from its list of dictionary words those words that are incompatible with the assumed assignment. After each iteration, the assignment probabilities and entropies of unprocessed $c_{i}$ 's are recomputed using the reduced lists of words.

\subsection{Ligatures and Partial Mappings}

The decoding procedure described above assumes each cluster ID maps to one output character, but some clusters actually contain ligatures and partial characters. To deal with partial characters, prior to the decoding steps described above, we count the number of times each subsequence of cluster IDs appears in the document. The subsequences that contain only $c_{i}$ 's that appear in no other subsequences are replaced by a single new cluster ID. To correct mapping errors that remain after the decoding step, we use a refinement strategy based on string-edit distances. The output alphabet is conceptually modified to $\Sigma^{\prime}=\Sigma^{*}$, the set of strings made of zero or more letters from $\Sigma$.

We begin with an example. Suppose we are given the partially decoded words

$$
\text { ?ost fri?tens enou? }
$$

where ? denotes the same cluster ID that needs to be deciphered. Recall that each cluster maps to an element of $\Sigma^{\prime}$, not necessarily to a single character. The first word alone does not give much information, since it can be cost, post, and almost, among others. From the second and third words, it becomes clear that the question mark stands for the letters $g h$. Essentially, this puzzle is solved by a knowledge of the English lexicon and a mental search for words that are most similar to those partial decodings.

The first step in this strategy is to identify the set $\tilde{C} \subset C$ of clusters that are candidates for correction. An intuitive definition of $\tilde{C}$ is the set of cluster IDs appearing only in non-dictionary words, but it misses those clusters appearing in decoded words that happen to be in the dictionary by accident. Instead, we define $\tilde{C}$ to be the set of clusters that occur more frequently in non-dictionary words than in dictionary words, where frequency is measured by the normalized character count.

For every decoded word $w_{i}$ that contains an element of $\tilde{C}$, we find the dictionary word that is closest to it in edit distance and tally the edit operations that involve elements of $\tilde{C}$. If $w_{i}$ happens to be in the dictionary, we count the identity mappings that involve elements of $\tilde{C}$. To avoid having to calculate the edit distance of $w_{i}$ to every dictionary word, we prune the list of dictionary words by computing the ratio $r\left(w_{i}, d_{j}\right)=\frac{\operatorname{comm}\left(w_{i}, d_{j}\right)}{\max \left(\left|w_{i}\right|,\left|d_{j}\right|\right)}$ for every dictionary word $d_{j}$, where $\operatorname{comm}\left(w_{i}, d_{j}\right)$ is the number of (non-unique) character trigrams that $w_{i}$ and $d_{j}$ have in common [5]. Let

\begin{tabular}{|ll|}
\hline aegean & aluvic \\
bernoulli & dlr \\
exxon & fluoroscan \\
multilaterally & zinn \\
\hline
\end{tabular}

Table 1. Some correctly deciphered nondictionary words from the ASCII code data.

$d\left(w_{i}\right)=\operatorname{argmax}_{d_{j} \in D} r\left(w_{i}, d_{j}\right)$, which can be found efficiently by using an inverted index of character trigrams. Next, only the string edit operations between $w_{i}$ and $d\left(w_{i}\right)$ need to be tallied. When multiple dictionary words share the same maximum ratio with $w_{i}$, the edit operations of $w_{i}$ are ignored, because empiracally, using such words skews the edit counts toward common letters such as e. After all edit counts have been tabulated, each cluster ID in $\tilde{C}$ is remapped to the string it most frequently edits to.

\section{Experiments and Analysis}

We performed experiments on artificial and real data. The lexicon used contains 10,683 words from the Spell Checker Oriented Word Lists (http://wordlist.sourceforge.net/).

Artificially-generated data provide a sanity check for the performance of the decoding algorithm under optimal conditions and allows us to examine the robustness of the algorithm by varying the amount of noise present. We use two types of artificial data in our experiments, one to simulate perfect character segmentation and clustering, and another to more closely resemble conditions of real-world images.

The best-case scenario for the decoding algorithm is when (1) there is a bijective mapping between clusters and the output alphabet $\Sigma$, and (2) the alphabet of the lexicon used by the decoder equals $\Sigma$. To simulate this condition, we clean data from the Reuters corpus by removing all digits and punctuation marks, and lowercasing all remaining letters. The three hundred files with the most words after preprocessing selected, and the ASCII codes of the text is given to the decoder. The number of words in these files range from 452 to 1046 . Table 2 shows the performance of the algorithm, and Table 1 lists some correctly decoded words that are not in the dictionary. Most errors involve mislabeling the letters $j$ and $z$, which make up $0.18 \%$ and $0.07 \%$ of the characters, respectively. In comparison, the letter e, which comprises $9.6 \%$ of the characters, was recalled $100 \%$ of the time.

Leetspeak (or Leet) is a form of slang used on the Internet that includes the substitution of letters by similar looking numerals (e.g., 3 for e), punctuation marks (e.g., $|-|$ for h), or similar sounding letters (e.g., ph for $f$ ). In addition, letter substitutions may vary from one occurence to another, 


\begin{tabular}{|llc|}
\hline & ASCII & Leetspeak \\
character accuracy & 99.80 & 99.65 \\
word accuracy & 98.84 & 98.06 \\
\hline
\end{tabular}

Table 2. Decoding performance on $\mathbf{3 0 0}$ news stories encoded in ASCII and Leetspeak.

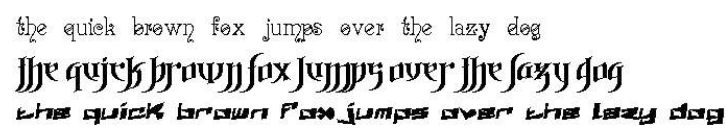

Figure 1. Examples of unusual fonts used to create document images of Reuters stories.

so that the letter s may be written as $\$$ in one word and 5 in the next. An example sentence in Reuters translated to Leetspeak is

! 7$\}-\{! n k$ it kind $0 \mathrm{ph}(\mathrm{V})$ udd! e $\$$ an @12e4dy mvddy $\$$ ! 7u@7! 0n

(i think it kind of muddies an already muddy situation).

Understanding Leetspeak requires solving some of the same issues in character recognition. More than one character in Leetspeak can be used to represent the same alphabet letter, which mirrors the problem of split clusters. Multiple Leet characters can be used to represent the same alphabet letter, and this mirrors the problem of over-segmentation of character images.

To generate Leetspeak data to test our decoding algorithm, we defined substitutions such that no two original letters share any characters in their mappings. This is done only as a simplification of the problem, since Leetspeak can be much more complex than what is presented here. We ran the decoding algorithm on the same 300 Reuters stories encoded in Leet, and Table 2 gives the character and word accuracies. The decoding performance on Leet is just as good as on the ASCII data with similar types of errors, so our algorithm seems to be robust to multiple representations of the same character and partial characters.

We evaluated our algorithm on two sets of images. The first consists of 201 Reuters news stories preprocessed in the manner described above and then rendered in unusual font styles (see Figure 1). These images are clean but do contain ligatures. The second set of images comes from the OCR data set of the Information Science Research Institute at UNLV [11], which includes manually-segmented text zones for each page. From a collection of Department of Energy reports in the UNLV data set scanned as bi-tonal images at $300 \mathrm{dpi}$, we picked 314 text zones that are primarily text (excluding zones that contain tables or math formulas) for recognition.

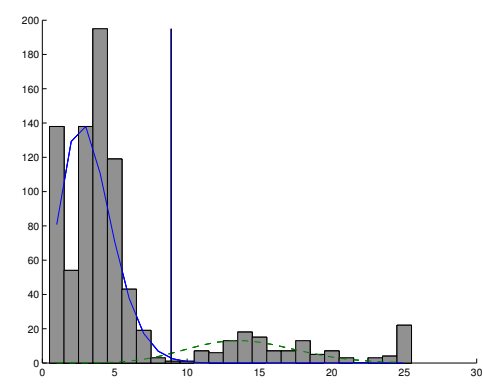

\section{Figure 2. A histogram of horizontal spacing gaps in an image. See text for details.}

Many of the images are slanted, where lines of text are not parallel to the top and bottom edges of the image. Although clustering can deal with slanted character images, rectification makes it easier to determine the reading order and inter-word spacing needed for decryption. Our rectification algorithm is based on an entropy measure of ink distributions. For each horizontal line of pixels in the image, we count the number of pixels occupied by ink to build a projection profile of the image as in [6]. We then search for the rotation that minimizes the projected entropy.

After rectification, the image is despeckled, and each connected component is extracted and resized to fit within a $60 \times 60$ pixel image centered at its centroid. To cluster the images, pairwise distances are computed by shifting one of the images around a $3 \times 3$ window and taking the smallest Hausdorff distance.

Our decoding algorithm relies on accurate segmentation of the sequences of cluster IDs into word units, so a principled method is needed to identify word demarcations. Figure 2 shows a typical histogram for horizontal spacing between adjacent connected components on an image, where the left hump corresponds to spaces within a word, and the right hump to spaces between two words. We model such histograms as mixtures of two Poisson distributions (the solid and dashed curves), one for intra-word spaces and another for inter-word spaces. The model contains a threshold $c$ (the vertical line) above which a horizontal spacing constitutes a word break.

Formally, the probability of a particular spacing $s_{i}$ is defined as

$P\left(s_{i} \mid c, \lambda_{1}, \lambda_{2}\right)$

$=P\left(s_{i} \in P_{1} \mid c\right) P_{1}\left(s_{i} \mid \lambda_{1}\right)+P\left(s_{i} \in P_{2} \mid c\right) P_{2}\left(s_{i} \mid \lambda_{2}\right)$

$=P\left(s_{i} \in P_{1} \mid c\right) P_{1}\left(s_{i} \mid \lambda_{1}\right)+\left(1-P\left(s_{i} \in P_{1} \mid c\right)\right) P_{2}\left(s_{i} \mid \lambda_{2}\right)$

$=I\left(s_{i}>c\right) P_{1}\left(s_{i} \mid \lambda_{1}\right)+\left(1-I\left(s_{i}>c\right)\right) P_{2}\left(s_{i} \mid \lambda_{2}\right)$,

where $I$ is the indicator function, and $P_{j}(j=1,2)$ are

Poisson distributions: $P_{j}\left(s_{i} \mid \lambda_{j}\right)=\frac{e^{-\lambda_{j}} \lambda_{j}^{s_{i}}}{s_{i} !}$.

Given the list of spaces $\left(s_{1}, \ldots, s_{N}\right)$, the objective func- 


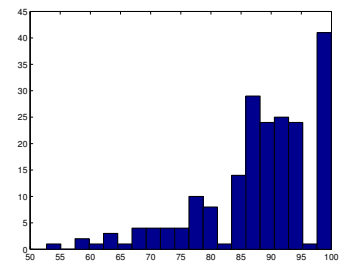

(a)

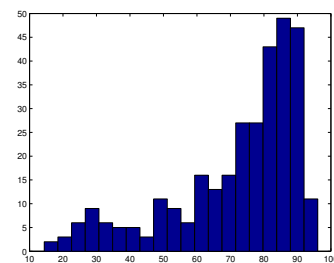

(b)
Figure 3. Histograms of character accuracies. (a) For 201 Reuters stories rendered in unusual fonts. (b) For 314 Dept. of Energy documents.

tion is simply defined by the likelihood of the data:

$$
\Omega\left(c, \lambda_{1}, \lambda_{2}\right)=\prod_{i=1}^{N} P\left(s_{i} \mid c, \lambda_{1}, \lambda_{2}\right) .
$$

The goal is to find the parameters $\theta=\left(c, \lambda_{1}, \lambda_{2}\right)$ that maximize $\Omega$. We do so using gradient ascent with the bold driver algorithm to adopt the learning rate at each iteration.

The indicator function is discontinuous so is not everywhere differentiable, which complicates the optimization routine. We avoid this problem by approximating $I$ by a shifted sigmoid function: $I\left(s_{i}>c\right) \approx \frac{1}{1+e^{c-s_{i}}}$.

The number of clusters is chosen using the "elbow criterion" heuristic: In each step of agglomerative clustering, the distance between the two clusters to merge is plotted, giving a curve that resembles the exponential function. The number of clusters to form is derived from the point where the slope of the curve begins increasing faster than some threshold value $\tau$. In our experiments, $\tau$ is manually set to 0.005 .

Figure 3 shows the histograms of character accuracies on the Reuters and UNLV test images. Averaged over the number of images, the mean character accuracy for the Reuters images is $88.09 \%$, and for the Dept. of Energy document it is $73.78 \%$. Limiting evaluation to lowercase characters gives a mean of $78.85 \%$. On the UNLV images, the mean accuracy for identifying word demarcations, averaged over the number of images, is $95.44 \%$. Although this figure initially looks promising, images with low accuracies are caused by unrecoverable errors in word segmentation. Our decoding algorithm also misses all digits, punctuation marks, and uppercase letters; this is an area for future work.

Similar to the results presented in [3], our unsupervised approach cannot recognize numerals, punctuation marks, or uppercase letters. Using image-to-character classifiers to identify those special characters beforehand proves beneficial, as discussed in [4]. To this end, we plan to combine cryptogram decoding with a robust maximum-entropy char- acter classifier used by Weinman and Learned-Miller [12].

We presented an unsupervised OCR system using character clustering with canopies and a cryptogram decoding algorithm based on numerization strings. Its performance was evaluated on artificial and real data. Under ideal input conditions, where both character segmentation and clustering are correct, our decoding algorithm correctly decodes almost all words, even those absent from the lexicon. Although our algorithm is not sufficient alone, it can improve the recognition performance of an OCR system when augmented with appearance models.

\section{Acknowledgments}

Supported by the Center for Intelligent Information Retrieval, and the CIA, NSA, and NSF under grants IIS0326349 and IIS-0546666.

\section{References}

[1] T. Breuel. Classification by probabilistic clustering, 2001.

[2] R. G. Casey. Text OCR by solving a cryptogram. In Int Conf. on Pattern Recognition, volume 86, pages 349-351, 1986.

[3] T. K. Ho and G. Nagy. OCR with no shape training. In Int. Conf. on Pattern Recognition, 2000.

[4] T. K. Ho and G. Nagy. Identification of case, digits and special symbols using a context window, 2001.

[5] K. Kukich. Technique for automatically correcting words in text. ACM Computing Surveys, 24(4):377-439, 1992.

[6] K. Laven, S. Leishman, and S. Roweis. A statistical learning approach to document image analysis. In 8th Int. Conf. on Document Analysis and Recognition, 2005.

[7] D.-S. Lee. Substitution deciphering based on HMMs with applications to compressed document processing. IEEE Trans. Pattern Anal. Mach. Intell., 24(12):1661-1666, 2002.

[8] D. A. Lisin, M. A. Mattar, M. B. Blaschko, M. C. Benfield, and E. G. Learned-Miller. Combining local and global image features for object class recognition. In Proc. of the IEEE Workshop on Learning in Computer Vision and Pattern Recognition, June 2005.

[9] A. McCallum, K. Nigam, and L. H. Ungar. Efficient clustering of high-dimensional data sets with application to reference matching. In Proc. of the sixth ACM SIGKDD Int. Conf. on Knowledge discovery and data mining, pages 169 178, 2000.

[10] G. Nagy. Efficient algorithms to decode substitution ciphers with applications to OCR. In Proc. of Int. Conf. on Pattern Recognition, 1986.

[11] T. A. Nartker, S. V. Rice, and S. E. Lumos. Software tools and test data for research and testing of page-reading OCR systems. In Int. Symp. on Electronic Imaging Science and Technology, 2005.

[12] J. Weinman and E. Learned-Miller. Improving recognition of novel input with similarity. In IEEE Conf. on Computer Vision and Pattern Recognition, 2006. 